\title{
Enquête sérologique sur la brucellose du dromadaire en Ethiopie
}

\author{
par J. DOMENECH (*)
}

\begin{abstract}
RÉSUMÉ
Une enquête sérologique sur la brucellose du dromadaire en Ethiopie montre un pourcentage de sérums positifs égal à 4,4 p. 100 si on prend un seuil de 30 UI par ml, ou de 0,9 p. 100 au seuil de 100 UI par ml.
\end{abstract}

\section{INTRODUCTION}

Dans le cadre d'une vaste étude sur l'élevage et la pathologie du dromadaire en Ethiopie, commencée en 1973, un certain nombre de sérums ont été collectés par le Dr D. RICHARD dans toutes les zones d'élevage camelin du pays (3).

Il a paru intéressant de rechercher quelle était l'importance de la brucellose, étant donné que le lait de chamelle tient une grande place dans l'alimentation des éleveurs nomades somali.

977 sérums ont donc été analysés, et les résultats sont présentés dans cette note.

\section{MATÉRIEL ET MÉTHODES}

Les 977 sérums ont été récoltés dans divers villages des provinces du Sidamo, du Harrar, du Tigre et de l'Erythrée.

$\mathrm{La}$ réaction de la séroagglutination lente en tubes, de Wright, a été effectuée avec un antigène agglutinant le sérum international ++ au 1/650 (antigène R. BELLON, France) $(1,2)$.

Les résultats sont notés positifs si on observe 50 p. 100 d'agglutination à la dilution finale du $1 / 20$ (30 UI) ou au-dessus, et douteux au $1 / 10$ (15 UI).

\section{RÉSULTATS. DISCUSSION}

(*) Veterinary Institute, Mission Vétérinaire Française en Ethiopie, P. O. Box 19, Debré-Zeit (Ethiopie).

Adresse actuelle: Laboratoire de Farcha, B. P. 433, N'Djamena (Tchad).

Les résultats sont exposés dans le tableau I.

TABLEAU $N^{\bullet}$ I - La brucellose du dromadaire en Ethiopie : enquête sérologique.

\begin{tabular}{|c|c|c|c|c|c|c|c|c|c|c|}
\hline \multirow{2}{*}{ Province } & \multirow{2}{*}{$\begin{array}{l}\text { Nombre } \\
\text { sérums }\end{array}$} & \multirow{2}{*}{ Nëgatif } & \multirow{2}{*}{ Douteux } & \multicolumn{5}{|c|}{ Positifs } & \multirow{2}{*}{$\begin{array}{c}\text { Total } \\
\text { positif }\end{array}$} & \multirow{2}{*}{$\begin{array}{c}\text { Pourcentage } \\
\text { positif }\end{array}$} \\
\hline & & & & $1 / 20$ & $1 / 40$ & $1 / 80$ & $1 / 160$ & $1 / 320$ & & \\
\hline Sidamo & 743 & 672 & 32 & 20 & 11 & 3 & 0 & 5 & 39 & 5,2 \\
\hline Harrar & 116 & 112 & 2 & 1 & 0 & 0 & 0 & 1 & 2 & 1,7 \\
\hline Ery three & 60 & 58 & 1 & 1 & 0 & 0 & 0 & $\mathfrak{o}$ & 1 & 1,6 \\
\hline Tigre & 58 & 50 & 7 & 1 & 0 & 0 & 0 & 0 & 1 & 1,7 \\
\hline Tatal & 977 & 892 & 42 & 23 & 11 & 3 & 0 & 6 & 43 & 4,4 \\
\hline
\end{tabular}


Sur 977 sérums analysés, 43 sont positifs à une dilution du $1 / 20$ ou plus, soit 4,4 p. 100 .

$\mathrm{Si}$ on choisissait comme seuil de positivité, la dilution du 1/80 (120 UI), le nombre de sérums positifs ne serait plus que de 9 , soit à peine 0,9 p. $100(2)$.

On peut donc conclure que la brucellose n'est pas un problème très important chez le dromadaire en Ethiopie. Cela recoupe les résultats des enquêtes faites auprès des éleveurs qui ne signalent pas les avortements contagieux comme très fréquents.

\section{CONCLUSION}

Avec un taux de positivité de 4,4 p. 100 , la brucellose ne parait pas être une composante majeure de la pathologie du dromadaire en Ethiopie.

\section{REMERCIEMENTS}

Nous remercions le Dr D. RICHARD qui a collecté les sérums analysés au Laboratoire, ainsi que les Dr M. VIGIER et J. FIKRE, directeurs du Veterinary Institute de Debre-Zeit, pour nous avoir permis de mener à bien cette étude.

\section{SUMMARY}

Serological survey of one humped camel brucellosis in Ethiopia

A serological suryey of one humped camel brucellosis in Ethiopia has shown that:

- with a positive of level of $30 \mathrm{UI}$ per ml, 4,4 p. 100 of the camels were positive :

- with a positive level of 100 UI per ml, only 0,9 p. 100 of the camels were positive.

\section{RESUMEN}

Encuesta serologica sobre la brucelosis del dromedario en Etiopia

Una encuesta serologica sobre la brucelosis del dromaderio en Etiopia ha mostrado un porcentaje de 4,4 p. 100 de positivos con un umbral de 30 UI por $\mathrm{ml}$, o de 0,9 p. 100 con un umbral de 100 UI por $\mathrm{ml}$.

\section{BIBLIOGRAPHIE}

1. ALTON (G. G.), JONES (L. M.). La brucellose. Techniques de Laboratoire. Genève, O. M. S., 1968 (Monographie ${ }^{\circ} 55$ ).

2. RENOUX (G.), GAUMONT (R.). Pathologie de la production du lait. II. Méthodes de diagnostic biologique des brucelloses animales. Cahiers Techniques du C. N. E. R. N. A., 1966.

3. RICHARD (D.). Etude de la pathologie du dromadaire dans la sous province du Borana (Ethiopie). Thèse. Doct. vét. Alfort, $1975, \mathrm{n}^{\circ} 75$. 